\title{
Sensitivity analysis of airline schedule optimization (ASO) advanced model
}

\author{
Danica Pavlovic* ${ }^{*}$ \\ Faculty of Transport and Traffic Engineering, University of Belgrade, \\ Vojvode Stepe 305, Belgrade, Serbia
}

\begin{abstract}
The ASO model provides a proposal for a new flight schedule, in situations when a carrier's flight scheduled is disrupted, and which would minimize the negative effects of the disruption. The objective function of the model is a mathematical formulation of the company's goals and preferences, where all assumptions and operational constraints must be satisfied. The disruption costs are given in weighted coefficients which present the penalties that can be changed by the dispatcher. One of the goals was to test to what degree the generated solutions are sensitive to changes in penalty values.
\end{abstract}

Keywords: sensitivity analysis, model, penalties, flight schedule, perturbation, airline profit

\section{I NTRODUCTI ON}

The daily schedule, handed to the dispatchers at the airline operations control centre in charge of observing its realization, is a set of aircraft and crew routings for a one-day time period. In addition to observing the daily schedule realization, dispatchers must deal with disruptions that may jeopardize the execution of airline's planned operations and cause flight delays and

\footnotetext{
* Danica Pavlović works as a teaching and research assistant at the Division of Aircraft Operations and Air Transport Planning and Management, Department of Air Transport at the Faculty of Traffic and Transport Engineering, University of Belgrade, Serbia, since October 2005. Major fields of interest: airline operations transport planning and modelling.

† E-mail: d.pavlovic@sf.bg.ac.yu, phone: +381 11 3091264, fax: +381 112496476
} 
cancellations, which may further incur direct and indirect operational costs to the company ${ }^{1}$. Taking all this into account, the successful running of the daily schedule is a very complex and demanding task for dispatchers.

Depending on the airline's policy, dispatchers at the operations control centre react to disruptions in different ways, but with the same goal: to minimize the negative effects on the realization of airline's planned operations. In order to minimize the negative effects of disruptions, the dispatcher is expected to create a solution and take appropriate action to implement the solution within a very short time period, i.e. in real time, such as flight delays, flight cancellations, substitution of aircraft in realization of certain flights, using spare aircraft from the fleet for realization of planned operations, etc. The complexity of the disruption problem increases when dispatcher has to deal with a larger number of flights and rotations.

This is why the need for computational methods and techniques arises. The implementation of these methods and techniques in the decision-making process should facilitate the creation of permissible and economical solutions within an acceptable period of time. This helps dispatchers in their work by accelerating the decision-making process and increasing the quality of decisions made.

An example of such software is the Airline Schedule Optimization (ASO) Advanced software which has been developed at The Faculty of Transport and Traffic Engineering, University of Belgrade (Institute of Faculty of Transport and Traffic Engineering, July 2002 - January 2005). The software is based on a model, and both (software and model) have the same goal: to provide a solution within a short period of time to create a new flight schedule in situations when the carrier's scheduled activities are disrupted, which would minimize the negative effects of the given disruptions.

\subsection{ASO ADVANCED SOFTWARE}

The ASO Advanced software is based on a mathematical model and heuristic procedure (Nedeljković, 2004). The proposed mathematical model and heuristic procedure are defined to calculate the objective function (the airline's profit of a new flight schedule) which is a

\footnotetext{
${ }^{1}$ Technical breakdown of aircraft, crew shortage, tardiness of crew and passengers, ATC constraints and restrictions, bad meteorological conditions, etc.
} 
mathematical formulation of the company's goals and preferences, where all assumptions and operational constraints must be satisfied. The aim of the objective function, and the number of assumptions and constraints taken into consideration in the model's process of creating solutions, depends on the user's preferences, hence it is possible to alter them, depending on the exact goal that is to be achieved.

The current version of the ASO Advanced software is based on the objective function of a mathematical model which maximizes the airline's profit and needs, and where all assumptions and operational constraints must be satisfied. The defined assumptions and operational constraints are based on different resources such as: number of aircraft, number of passenger seats, airport working hours, maximum allowed delay time, etc.

The objective function of the ASO Advanced model is determined in such a way that in the case of disruption, it maximizes the difference between revenues from sold passenger tickets on all flights and pondered costs that include: direct and indirect operational costs of assigning aircraft to flights, priority flight cancellation costs, flight delay costs, non-priority flight cancellation costs, aircraft maintenance disruption costs and aircraft balance disturbance costs (if at the end of day the aircraft are not at the planned airports).

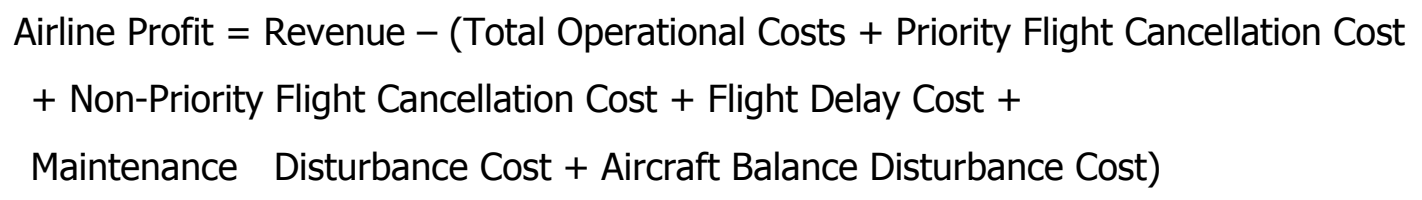

The assumptions and constraints significant for this particular research, among others, are: the airline has different types of aircraft in its fleet (different seat capacities), ferry flights (flights without passengers) are not allowed, crew constraints are not considered, all flights have to be executed within the period when the airports are open, etc.

To attain solutions related to the company's interests by using the ASO Advanced model, i.e. to avoid unacceptable solutions, there are several penalties introduced in the model's objective function, and values and relations between them can also be changed, according to defined goals. Those penalties are: 
$\rightarrow \underline{\mathrm{k}}_{1}-$ penalty for flight delay (cost per minute of delay)

$\rightarrow \underline{k}_{2}$ - penalty for non-priority flight cancellation (cost per flight)

$\rightarrow \underline{k}_{3}$ - penalty for aircraft maintenance disturbance (cost per flight)

$\rightarrow \underline{\mathrm{k}(\mathrm{i})}$ - penalty for priority flight cancellation (cost per flight)

$\rightarrow \underline{\operatorname{kaz}(l, k)}$ - penalty for aircraft balance disturbance (cost per flight)

The value of these penalties is very difficult to measure and quantify, but their values and relationships have an important influence on what the final solutions will be. According to these, the most important task is to carefully determine the values of penalties so the proposed solutions by the ASO Advanced model are in accordance with the company's goals and preferences. Changing their values could lead to a change in the solutions generated by the ASO Advanced model.

It is important to notice that the value of the objective function (the airline's profit) is not significantly representative when validating the quality of the generated solution. The quality of the generated solution is measured by the number of cancelled flights, total delays, single flight delay, etc. To improve the software to present the real profit of an airline for the proposed solution (new flight schedule) it is necessary to provide software with complete and precise data.

The main task in this paper is to analyze how the values of the penalties influence the solutions generated by the ASO Advanced model i.e. how sensitive the generated solutions are to changing the penalties values. Also, it was possible to determine the range of penalty intervals within the solution is the same. Because of the nature of the problem, the entire analysis and all the solutions and conclusions are based on a concrete example and should be viewed in that way. In another words, every flight brings different profit to an airline so the penalty value will have different influence on them i.e. on generated solutions proposed by the model. In any case, this analysis can help attain at least approximate values of the penalties which can further help achieving the compliance of the proposed solutions with the company goals and preferences. 


\section{SENSITIVITY ANALYSIS}

The sensitivity analysis is usually conducted after the determination of optimal solution, but if all the inputs are defined in advance it is possible to do it beforehand. The ASO Advanced model sensitivity analysis is tested on the flight schedule of a middle size European airline and all the flight data is known, with the exception of the values of the penalties which were estimated according to the policy of this particular airline.

For the purpose of sensitivity analysis, the basic solution was determined and accepted basic values of penalties were:

$$
\begin{aligned}
& +\mathrm{k}_{1}=1 \\
& +\mathrm{k}_{2}=10000 \\
& +\mathrm{k}_{3}=5000 \\
& +\mathrm{k}(\mathrm{i})=20000 \\
& +\mathrm{kaz}(\mathrm{l}, \mathrm{k})=3000
\end{aligned}
$$

Furthermore, it is defined that the maximum allowed flight delay is 300 minutes, according to the rules of the observed airline. This paper does no not consider how changing this value would influence the generated solutions.

For better understanding the model it is important to note that the first step in the algorithm is common for all solutions. In this step the model checks what kind of perturbation is in question, how many flights are affected and what type of flights they are (priority or non-priority). At the end of the first step, the model compares the maximum allowed flight delay with the delay of each affected flight, if those flights are going to be executed by the planned aircraft after the perturbation is removed. If the delay is longer then the maximum allowed delay for a given flight, that flight will be temporary cancelled. In the given example the delays of all affected flights are longer than the maximum allowed delay i.e. 300 minutes, so all the affected flights will be temporary cancelled. According to this, the penalties values have no influence on affected flights, in this first step. The only influence that they have is how the perturbation can be solved i.e. what the proposed solutions will look like. 
In further steps, the model is checks whether the temporary cancelled flights can be assigned to some other aircraft in the fleet, complying with all defined assumptions and operational constraints. For each provided solution the airline's profit is calculated and all solutions are then ranked in descending order. The list of the feasible solutions is then presented to the user.

Further in the paper it will be presented how the airline's profit and proposed solutions vary by varying the value only one penalty while the rest of the penalties keep their basic values, the range of penalty intervals within the solution is the same and finally, limited values for each of the penalties which should not be exceeded to avoid an unacceptable solutions, in this case the solutions which do not comply with the policy of the observed airline. After the one-penalty variation sensitivity analysis, some of the results gained from the two-penalty variation sensitivity analysis will be presented, where now two of five penalties are varyed while the rest of the penalties keep their basic values.

\subsection{EXAMPLE}

The data used in this research is the real data from realized operations in the daily schedule of the observed airline during a one-week time interval (April 17-30, 2006). The data required for testing was made available thanks to the Information Technology Department of observed airline. The daily flight schedule is presented on the Figure 1.

Example. Technical breakdown on the aircraft B737-G, registration OELNO, from 5:00 AM till 5:00 PM, at Vienna airport:

o Disruption duration equals to $12 \mathrm{~h}$, i.e. $720 \mathrm{~min}$.

o Affected flights by disruption:

- Priority flights: OS301 (VIE-CPH) and OS302 (CPH-VIE),

- Non-priority flights: OS313 (VIE-ARN) and OS314 (ARN-VIE). 


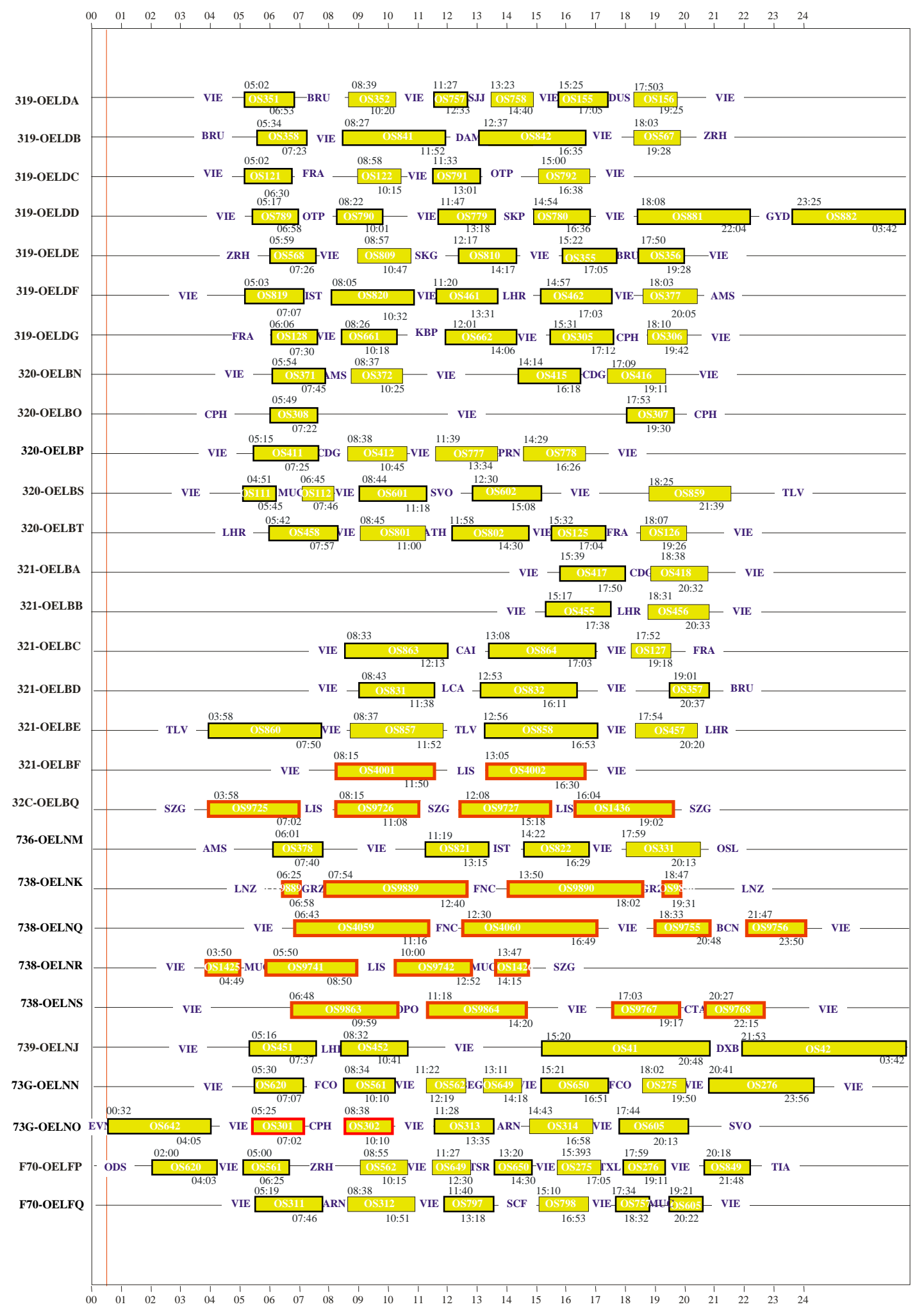

Figure 1. Daily flight schedule 


\section{Basic solution:}

- Priority flights, OS301 (VIE-CPH) and OS301 (CPH-VIE), are reassigned to aircraft A319 - OELDB and are executed with delay (delay on flight OS301 is $163 \mathrm{~min}$ and $112 \mathrm{~min}$ on flight OS302)

- Non-priority flights originally assigned to aircraft OELDB (OS841, OS842 and OS567), are executed with delay (delay on flight OS841 is $260 \mathrm{~min}$, delay on flight OS842 is 260 min and delay on flight OS567 is $217 \mathrm{~min}$ )

- Non-priority flights OS313 (VIE-ARN) and OS314 (ARN-VIE) are reassigned to the aircraft A320- OELBO and executed on time.

- No cancelled flights.

- $\quad$ The airline's profit is $\mathbf{F}=\mathbf{1 3 9 6 3 2 . 6 3}$

\subsection{ONE-PENALTY VARI ATI ON SENSI TI VITY ANALYSIS}

\subsubsection{PENALTY FOR PRI ORITY FLI GHT CANCELLATI ON, $k(i)$}

After the basic solution where $k(i)=20000$, the model was tested for a solution if this penalty is equal to zero i.e. if there is no penalty for cancelling the priority flight. Further, the analysis of the number of different solutions by varying the value of $k$ (i) was carried out and also, the values when the solution is changed were determined. The values of $k(i)$ used in the analysis and the airline's profit gained from the solutions as well as the solution reviews are presented in the following Table 1.

Table 1 The airline's profit and solution reviews for different values of $k(i)$

\begin{tabular}{|c|c|c|c|}
\hline Penalty k(i) & Airline's profit & Proposed solution & \\
\hline 0 & 142964,03 & $\begin{array}{l}\text { - Priority flights OS301 and OS302 are cancelled. } \\
\text { - Non-priority flights OS313 and OS314 are reassigned to the aircraft A320- } \\
\text { OELBO and are executed on time. }\end{array}$ & $\begin{array}{l}\bar{n} \\
\frac{0}{5} \\
\frac{\overline{0}}{2}\end{array}$ \\
\hline 1700 & 139632,63 & Basic solution & \\
\hline
\end{tabular}


The results of analysis show that varying the value of the $k(i)$ the airline's profit is also varying, but only until the airline's profit reaches the value of $F=139632.63$. After that the airline's profit remains constant despite that the value of the $k(i)$ continues to vary. The results also show that varying the value of the $k(i)$ there could be only two types of solutions for this particular example. The difference between them is in the number of cancelled flights, which exists in the first solution but not in the second one. Table 1 shows that while the penalty value increases, the airline's profit decreases, which was expectable considering that the penalty cost for each cancelled flight was increasing. The airline's profit decreases down to its minimum i.e. until $k(i)$ reaches the value when the penalty cost for cancelled flights is higher then total delay cost for all flights that aircraft A319 (OELDB) should execute in accordance with the proposed solution. The value of $k(i)$ when the solution changing occurs (from solution I to basic solution) is $\mathrm{k}(\mathrm{i})=1666$. This is also the lower limit of the $k(i)$ and if a user wants to avoid unacceptable solutions i.e. the solutions with cancelled priority flights, the values for $k(i)$ should not be below this limit.

Figure 2 shows that the airline's profit is very sensitive to varying the value of $k(i)$ but only in the first segment, until the $k(i)$ reaches the value of $k(i)=1666$. After this value the airline's profit is totally insensitive to varying the value of $k(i)$.

Figure 2. The airline's profit in regard to the value of $k$ (i) and the point of solution replacement

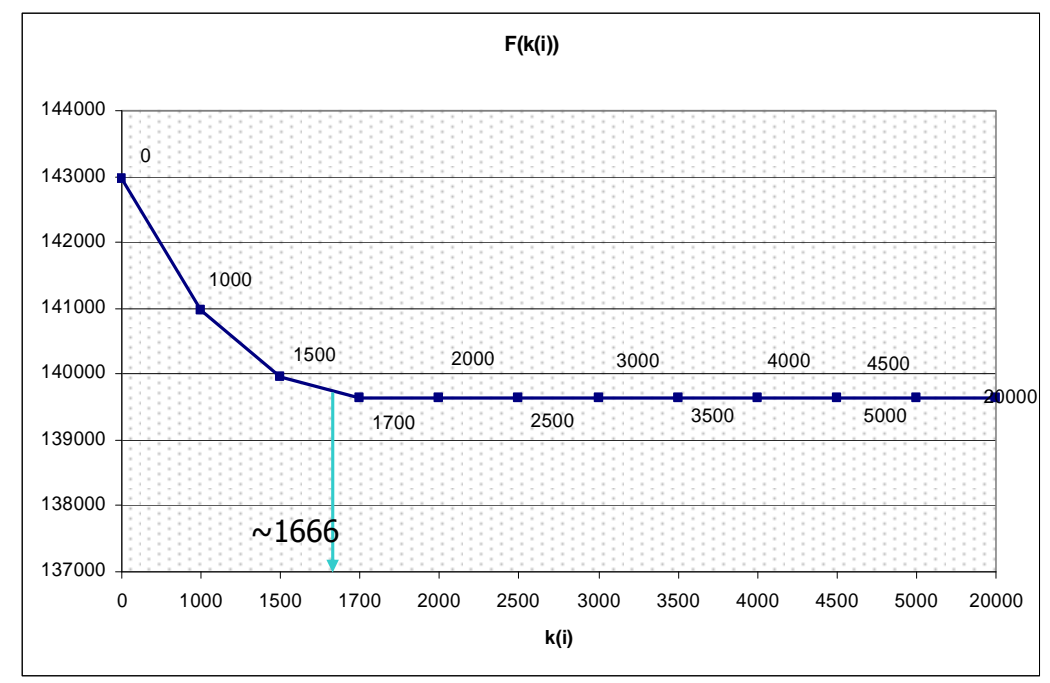




\subsubsection{PENALTY FOR NON-PRIORITY FLIGHT CANCELLATION, $\mathrm{k}_{2}$}

The basic solution is based on the value of $k_{2}=10000$, so as in the previous case, the solutions were generated first for the $k_{2}=0$ and then for the higher values. The solution reviews are presented in Table 2.

The results from Table 2 show that if this penalty would not exist or $k_{2}=0$, the airline's profit would be $F=167042.96$ and this value is considerably higher than the value of the airline's profit when there is no perturbation in the observed flight schedule $(F=140350.91)$.

Table 2 The airline's profit and solution reviews for different values of $k_{2}$

\begin{tabular}{|c|c|c|c|}
\hline Penalty $k_{2}$ & $\begin{array}{l}\text { Airline's profit } \\
\text { F }\end{array}$ & Proposed solution & \\
\hline 0 & 167042,96 & $\begin{array}{l}\text { - Priority flights OS301 and OS302 are reassigned to aircraft A319 (OELDD) } \\
\text { and are executed on time. } \\
\text { - Non-priority flights OS313 and OS314 are reassigned to aircraft A319 } \\
\text { (OELDF) and are executed on time. } \\
\text { - Non-priority flights originally assigned to aircraft OELDD (OS789, OS790, } \\
\text { OS779, OS780, OS881 and OS882) and OELDF (OS819, OS820, OS461, } \\
\text { OS462 and OS377) are cancelled. }\end{array}$ & 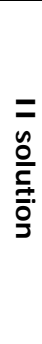 \\
\hline 1000 & 158109,02 & $\begin{array}{l}\text { - Priority flights OS301 and OS302 are reassigned to aircraft A319 } \\
\text { (OELDD) and are executed on time. } \\
\text { - Non-priority flights OS313 and OS314 are cancelled. } \\
\text { - Non-priority flights originally assigned to aircraft OELDD (OS789, OS790, } \\
\text { OS779, OS780, OS881 and OS882) are cancelled. }\end{array}$ & 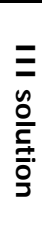 \\
\hline 3000 & 143252,07 & $\begin{array}{l}\text { - Priority flights OS301 and OS302 are reassigned to the aircraft A319 } \\
\text { (OELDB) and are executed with delay (delay on flight OS301 is } 163 \mathrm{~min} \text {, } \\
\text { and } 112 \mathrm{~min} \text { on flight OS302). } \\
\text { - Non-priority flights originally assigned to aircraft OELDB (OS841, OS842 } \\
\text { and OS567), are executed with delay (delay on flight OS841 is } 260 \mathrm{~min} \text {, } \\
\text { delay on flight OS842 is } 260 \text { min and delay on flight OS567 is } 217 \mathrm{~min} \text { ). } \\
\text { - Non-priority flights OS313 and OS314 are cancelled. }\end{array}$ & \\
\hline 5000 & 139632,63 & Basic solution & \\
\hline
\end{tabular}

However, if this penalty would not exist, the main problem would not be in the value of the airline's profit but in the proposed solution. Namely, all the flights that are originally assigned to the aircraft with registration OELDD and OELDF are cancelled after the perturbation occurs and 
after the affected flights are reassigned to these aircraft. The total number of cancelled flights is 11 (see Table 2 above).

Additionally, the balance in aircraft numbers at individual airports at the end of the day is disturbed because the aircraft OELDF finished its last rotation at the Vienna airport instead at the Amsterdam airport. The rotation VIE-OTP-VIE, originally assigned to the aircraft OELDD, is justifiable cancelled because the maximum allowed delay constrain is not satisfied. The rest of the cancelled flights could be executed on time or with acceptable delay, but still they were cancelled. The explanation of the reason why the software offered such a solution and what criteria were decisive lie in the way how the solutions are generated through the steps of algorithm.

Namely, when in perturbation, the affected flights are the priority flights and delay is longer then maximum allowed delay, the first solution is generated by reassigning the affected priority flights to some other aircraft, if the given conditions are satisfied (the aircraft is at the same airport as broken one or it is going to arrive at the same airport, the aircraft capacity is larger or equal to the number of passengers from affected flights, etc). Additionally, that aircraft has to be possible to execute the affected priority flights and the rest of the flights from their mini rotation and after that, to continue to execute the flights that are originally assigned to it. If there is more then one aircraft that can execute the affected priority flights, the one with least cancelled flights caused by the execution of priority flights will be selected. If there is more aircraft with the same, minimum number of cancelled flights, the one with minimum overall delay will be selected. If there is more aircraft with the same overall delay, the one, first found, will be selected.

When the first solution is determined, the next step is to reassign the rest of the temporary cancelled flights to the aircraft that can execute them on time or with an allowed delay. Otherwise, those flights stay cancelled till the end of the day.

The above describe procedure for solving the flight schedule perturbation was also applied in the case when the value of $k_{2}$ was 0 . In that case, when the affected priority flights were 
reassigned to the aircraft OELDD, the rest of the flights, originally assigned to this aircraft, were temporary cancelled because each flight would exceed the allowed delay if they were executed after the priority flights. In the next step these temporary cancelled flights were returned to flight schedule and then it appeared that because there was no penalty for cancelling the nonpriority flights $\left(k_{2}=0\right)$, the airline's profit would be higher if these flights were cancelled. The main reason was that the direct operational costs of that aircraft was higher then the realized revenue from these flights.

However, the airline policy is that all planned flights must be executed no matter if they are profitable or not, so the solution where the flights are cancelled just because they are not profitable is unacceptable. According to this, all the values of $k_{2}$ greater then 4810 would help to avoid the unacceptable solution.

To avoid the similar situations in other perturbations, the model should be improved by adding one more constraint that would protect the unprofitable flights.

Figure 3 The airline's profit in regard to the value of $k_{2}$ and the points of solution replacement

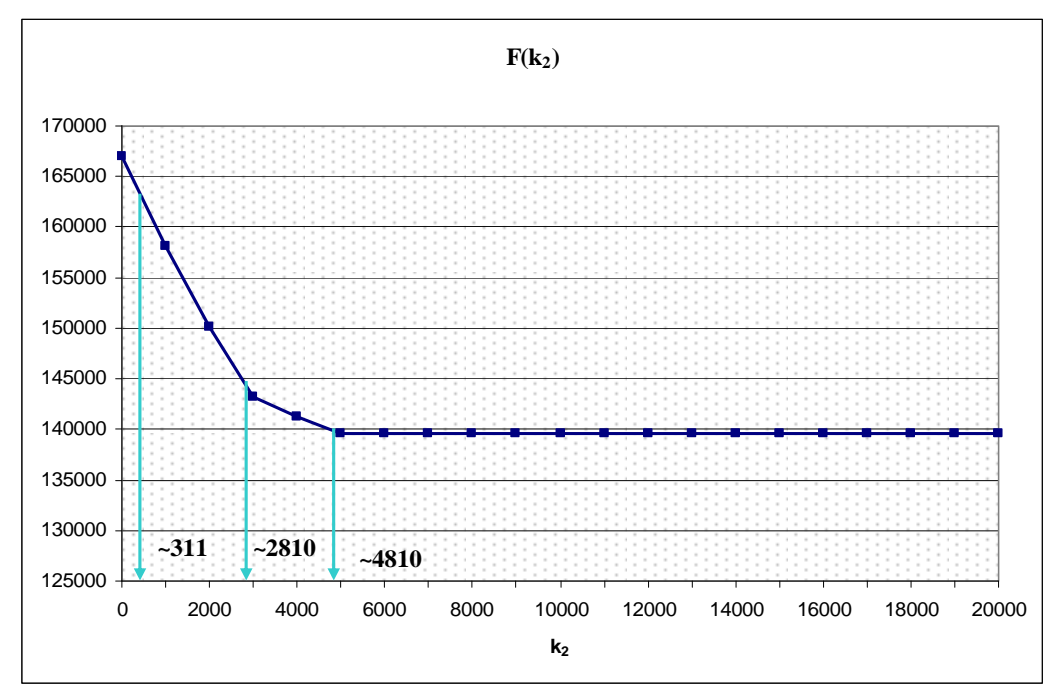

Figure 3 shows that the greater sensitivity of the airline's profit by varying the value of $k_{2}$ is in the first segment when the value of $k_{2}$ is smaller then 4810 , after that the airline's profit is totally insensitive to varying the value of $k_{2}$. Also, in the first segment the airline's profit is not sensitive in the same way for all the values of $k_{2}$. In the first interval from 0 to 2810 , the 
function slope is inclined i.e. the function value decreases rapidly for small changes in the $k_{2}$ value. In the second interval from 2810 to 4810 the airline's profit sensitivity is smaller and the slope is less inclined.

\subsubsection{PENALTY FOR FLI GHT DELAY, $\mathbf{k}_{1}$}

In the basic solution, this penalty has the value $k_{1}=1$, so the first analysis is done for the case when this penalty is not considered i.e. $k_{1}=0$, and then its value is increasing. The next Table 3 shows the generated solutions for different values of $k_{1}$ and it can be noticed that overall delay is reduced as well as the number of delayed flights with increasing the value of $k_{1}$.

Table 3 The airline's profit and solution reviews for different values of $k_{1}$

\begin{tabular}{|c|c|c|c|}
\hline Penalty $k_{1}$ & $\begin{array}{c}\text { Airline's profit } \\
\text { F }\end{array}$ & Proposed solution & \\
\hline 0 & 140644,63 & Basic solution & \\
\hline 2 & 139068,31 & $\begin{array}{l}\text { - Flights OS301, OS302, OS313 and OS314 are reassigned to aircraft A320 - } \\
\text { OELBO and are executed with delay (delay on flight OS301 is } 162 \mathrm{~min} \text {, } \\
\text { delay on flight OS302 is } 111 \mathrm{~min} \text {, delay on flight OS313 is } 78 \mathrm{~min} \text { and delay } \\
\text { on flight OS314 is } 55 \mathrm{~min} \text { ). } \\
\text { - Non-priority flight OS307 is reassigned from OELBO to aircraft A320 - } \\
\text { OELBP and is executed on time. }\end{array}$ & $\begin{array}{l}\frac{1}{5} \\
\frac{0}{5} \\
\frac{0}{5}\end{array}$ \\
\hline 3 & 138939,51 & $\begin{array}{l}\text { - Priority flights OS301 and OS302 are reassigned to aircraft A321 - OELBA } \\
\text { and are executed on time. } \\
\text { - Non-priority flights OS313 and OS314 are reassigned to aircraft A320 - } \\
\text { OELBO and are executed on time. }\end{array}$ & $\begin{array}{l}\leq \\
\text { 을 } \\
\text { 흉 }\end{array}$ \\
\hline
\end{tabular}

Further, for $k_{1}=3$ the model proposed a new solution where all affected flights are executed on time. The proposed flight distribution to available aircraft can be considered as "optimal" from the aspect of delay and cancellation cause in the situation, where one aircraft is out of order 12 hours and where the possibility of cancelling certain flights exists, still there is a solution where all planned flights can be executed on time with the available fleet. On the other side, it has to be taken into consideration that for execution of the priority flights, OS301 and OS302, the 
aircraft with higher direct operational costs is used, so it now depends on airline policy if the generated solution for $k_{1}=3$ is going to be acceptable or not.

As expected, further increasing the $k_{1}$ value does not have any influence on the airline's profit or on generated solutions, because any $k_{1}$ value equal or higher then 3 would exceed the costs of using the more expensive aircraft then the broken one.

Figure 4 The airline's profit in regard to the value of $k_{1}$ and the points of solution replacement

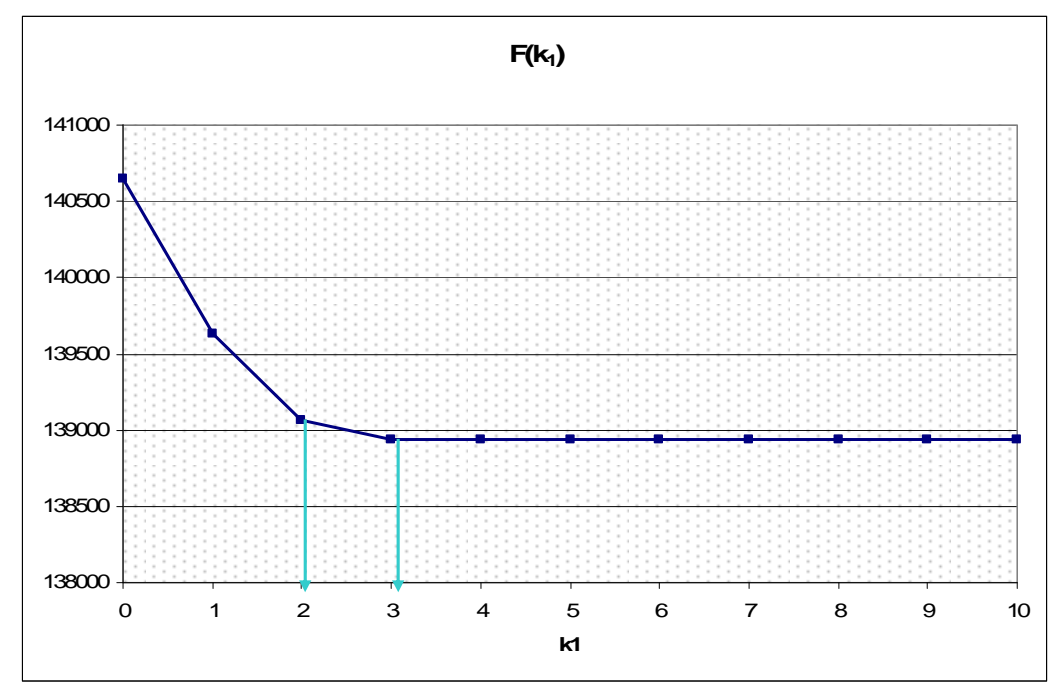

The Figure 4 shows that the airline's profit is very sensitive to varying the value of $k_{1}$ in the segment where the $k_{1} \leq 3$, after this value the airline's profit is totally insensitive to varying the value of $k_{1}$.

\subsubsection{PENALTY FOR AI RCRAFT BALANCE DISTURBANCE, $k a z(l, k)$}

The airline has some additional costs if the balance in aircraft numbers at individual airports at the end of the day is disturbed. For that reason in this model the penalty for aircraft balance disturbance, $k a z(l, k)$, is introduced and because this cost is very hard to measure the sensitivity analysis for this penalty is also done. 
The starting value in the basic solution is $\operatorname{kaz}(\mathrm{l}, \mathrm{k})=3000$ but the results from the sensitivity analysis shows that the value of $k a z(l, k)$ has no influence on the airline's profit and generated solution for the observed example. One of the reasons is that the affected flights are early in the morning so the possibility that the perturbation is going to be extended until the end of the day is very low. Also, the reason why this penalty has no greater influence on the generated solutions in this example is that the entire analysis is done according to basic solution i.e. according to basic penalty values where the situation in which the penalty $k a z(l, k)$ would be considered does not appear. In another words, there is no aircraft balance disturbance in the observed example.

That is why the additional test was done which proved that penalty $k a z(l, k)$ has influence on generated solutions. According to this results with the values higher then $\operatorname{kaz}(l, k)=3700$ the unacceptable solutions can be avoided.

\subsubsection{PENALTY FOR AI RCRAFT MAI NTENANCE DISRUPTION $k_{3}$}

Finally, the last parameter $k_{3}$ is the penalty for aircraft maintenance disruption i.e. the additional airline cost if aircraft is not at the airport where its maintenance is planned. The starting value of this penalty was also estimated according to the policy of the observed airline, but $k_{3}$ was not the subject of sensitivity analysis.

First of all, there were some difficulties in getting the information on scheduled maintenance of aircraft from the observed airline's fleet. Later it transpired that aircraft maintenance demands are very flexible so that they do not represent a constraint of major importance to the dispatchers' solution creating process. That is: dispatchers easily make arrangements with the personnel in the Maintenance Department related to moving scheduled aircraft checks, in cases where they need particular aircraft for the realization of scheduled operations.

Based on this, one could conclude that the penalty $k_{3}$ has no great importance in the ASO Advanced model usage. However, the importance of this penalty should not be underestimated overall or when applying it to the flight schedule of another airline with different airline' policy. 


\subsection{TWO-PENALTY VARI ATION SENSITIVITY ANALYSIS}

Based on analysis so far, it can be concluded that the solutions generated by the ASO Advanced model depends on certain factors such as: penalty values, number of passengers on flights, passenger ticket price, maximum allowed flight delay, etc. All these factors, except the passenger ticket price, are variables whose values are very difficult to estimate, but the most significant influence on the model's solutions and the most difficult to determine are the penalty values. The previous sensitivity analysis was useful for gaining the recommended value for each penalty that would lead to solutions that are logical and expectable.

Even without precise determination of penalties values, important questions that need answers are whether varying the value of one penalty will this influence on the value of another penalty, in what way and to what extent. Based on this, the following analysis was done where the values of two penalties were varying simultaneously. Two-penalty variation sensitivity analysis covered all combinations of penalties but in this paper only the one with most important results will be presented.

\subsubsection{PENALTY FOR PRIORITY FLIGHT CANCELLATION k(i) AND PENALTY FOR FLIGHT DELAY $\mathbf{k}_{1}$}

The results from the analysis where the values of two penalties are varied, the penalty for priority flight cancellation value $(\mathrm{k}(\mathrm{i}))$ and penalty for flight delay value $\left(\mathrm{k}_{1}\right)$, show:

how the solutions change

A how the point of solution replacement changes

how the airline's profit changes

In the two-penalty variation sensitivity analysis of the ASO Advanced model, the first result is the solution where both penalties have values zero, and after that the value of $k(i)$ increases while the value of $k_{1}$ remain zero. This is phase 1 and for this phase the following results are determined: changing of airline's profit as well as the solution changing until airline's profit 
reaches the final value ${ }^{2}$ for given parameters. The same procedure was repeated as many times as it was needed and in each next phase the value of $k_{1}$ was increased by 1 .

The common element for all phases is that when $k(i)=0$ the solution is always the same regardless of the value of penalty $k_{1}$. The structure of that solution is:

A priority flights OS301 and OS302 are cancelled;

A non-priority flights OS313 and OS314 are reassigned to the aircraft A320 - OELBO and are executed on time.

Also, the common element for all phases is that each phase has an equal number of solutions during the variation of $k(i)$, and that number is 2 . The first one, as mentioned above, is common for all phases and the second one depends on the phase.

Figure 5 presents the point where it comes to solution changing (when the ASO Advanced software starts to propose the second instead of first solution) and how the value of this point $(k(i))$ is changing in regards to changing the value of penalty $k_{1}$.

Figure 5 Changing the point of solution replacement in regards to changing the value of penalty $k_{1}$

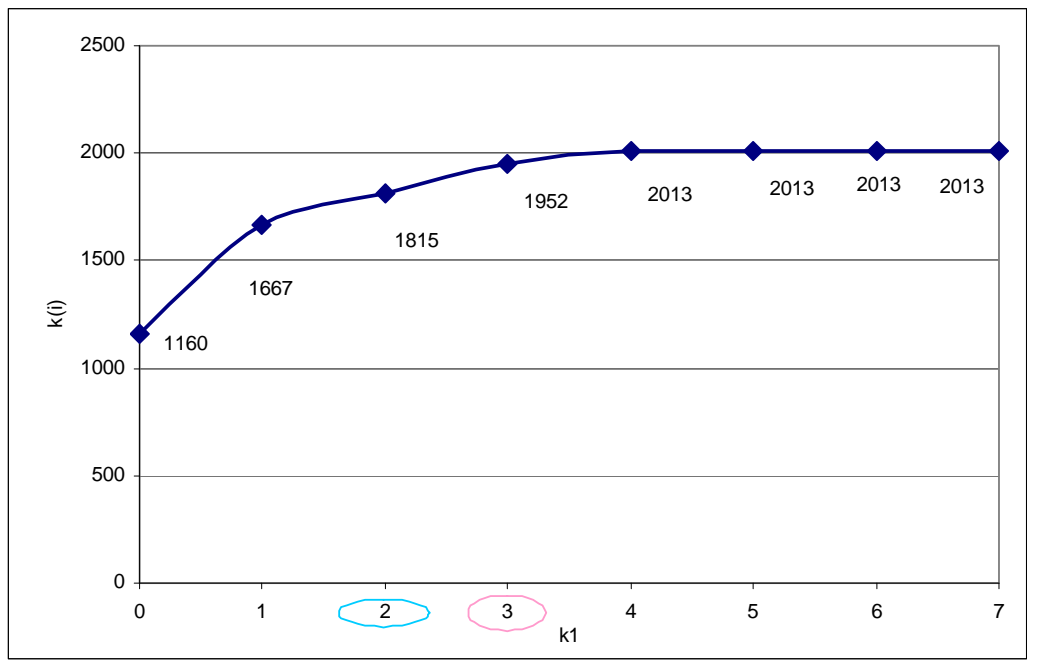

${ }^{2}$ When airline's profit reaches this value it stays constant despite the further penalty value changing. 
Figure 5 shows that by increasing the value of penalties, $k(i)$ and $k_{1}$, the point of solution replacement takes a higher value. Also, it can be noticed that the value of this point is increasing until the airline's profit reached the value of 2013, but for higher values it is constant. The marked values of $k_{1}$ on Figure 5 represent the values when the second solution differs from the second solution in previous phase. This happens for $k_{1}=2$ and $k_{1}=3$ and according to this there are 3 types of second solutions that appear in the analysis where the values of two penalties, $k(i)$ and $k_{1}$, vary. These 3 types of solutions are presented in Table 4.

Table 4 The presentation of II solution

\begin{tabular}{|cc|}
\hline Penalty $\mathbf{k}_{\mathbf{1}}$ & II solution \\
\hline 0 & Basic solution \\
\hline 2 & V solution \\
\hline 3 & VI solution \\
\hline
\end{tabular}

By increasing the value of $k_{1}$, the number of delayed flights decreases, until the proposed solution has no delayed or cancelled flights i.e. until the delay cost exceeds the costs of using the more expensive aircraft then the broken one. Figure 5 and Table 4, also, show that for gaining the "optimal" solution the $k_{1}$ value should not be less then 3 , and the $k(i)$ value should not be less then 1952, which is by 286 a higher value than the one determined in previous analysis (1666).

Figure 6 presents the airline's profit for different values of penalties, $k(i)$ and $k_{1}$. According to these results, while the values of $k(i)$ and $k_{1}$ increases, the value of airline's profit decreases down to its minimum and after that is constant. It can be concluded that airline's profit is very sensitive between 0 and 4 for $k_{1}$ value and between 0 and 2000 for $k$ (i) value. For higher values then this one the airline's profit is insensitive. The Figure 6 best describes how the value changing of $k_{1}$ influence on the ASO Advanced model sensitivity. Meaning, that increasing the value of $k_{1}$ will increasingly decrease the airline's profit, i.e. the airline's profit will have lower minimum and it will reach that minimum at higher values of $k(i)$. The penalty values of $k_{1}$ have no influence on airline's profit at the values of $k(i)<1200$ because of the solution structure where the flights are executed on time or cancelled. There are no delayed flights. 
Figure 6 The airline's profit regarding to penalties values, $k(i)$ and $k_{1}$

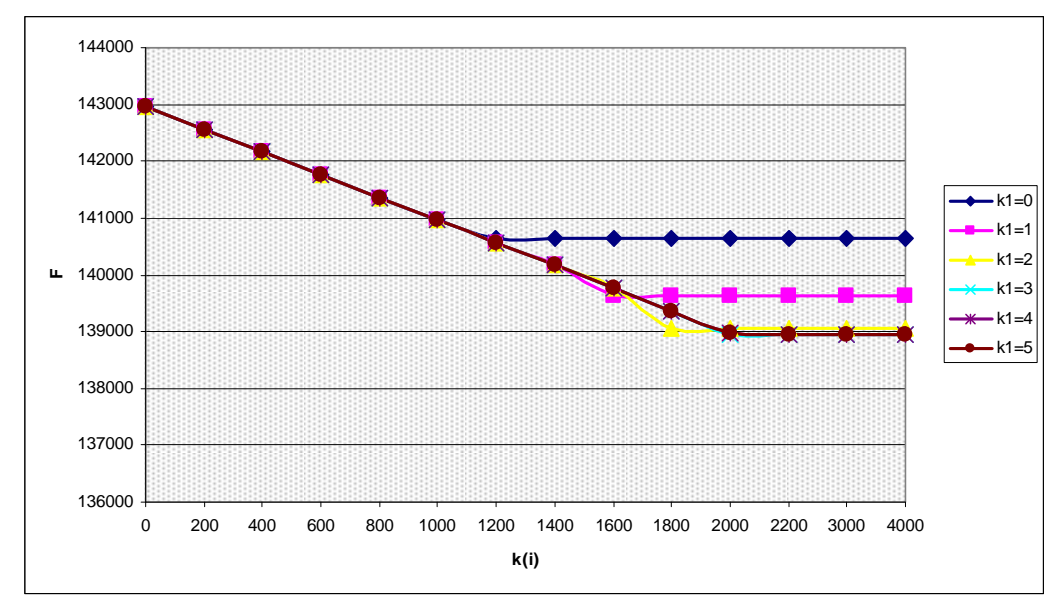

\subsubsection{PENALTY FOR NON-PRI ORITY FLI GHT CANCELLATION $k_{2}$ AND PENALTY FOR FLI GHT DELAY $\mathbf{k}_{1}$}

As in previous case, the same procedure was applied on the second pair of penalties $k_{1}$ and $k_{2}$. The common for all phases is that in each phase the overall number of different solutions is 4 . Also, in each phase when $k_{2}$ is 0 the solution is always the same regardless of the value of penalty $k_{1}$ and the model keeps this solution until penalty $k_{2}$ reaches the value of 312 . After this value the model is proposing the second solution and this solution is also common for all phases. The structures of mentioned solutions are:

I solution:

o Priority flights OS301 and OS302 are reassigned to aircraft A319 (OELDD) and are executed on time.

o Non-priority flights OS313 and OS314 are reassigned to aircraft A319 (OELDF) and are executed on time.

o Non-priority flights originally assigned to aircraft OELDD (OS789, OS790, OS779, OS780, OS881 and OS882) and OELDF (OS819, OS820, OS461, OS462 and OS377) are cancelled.

II solution:

o Priority flights OS301 and OS302 are reassigned to aircraft A319 (OELDD) and are executed on time.

o Non-priority flights OS313 and OS314 are cancelled. 
o Non-priority flights originally assigned to aircraft OELDD (OS789, OS790, OS779, OS780, OS881 and OS882) are cancelled.

Figure 7 below shows the point where the solution change occurs and how the value of this point $\left(k_{2}\right)$ changes in regards to the change of the value of penalty $k_{1}$. According to the fact that each phase has 4 solutions, Figure 7 presents the value of penalty $k_{2}$ when the change occurs in the solution from I to II, from solution II to III solution and, finally, from III to IV solution. Additionally, it is marked when the structure of solutions III and IV changes while increasing the value of $k_{1}$

Figure 7 Changing the point of solution replacement regards to changing the value of penalty $k_{1}$

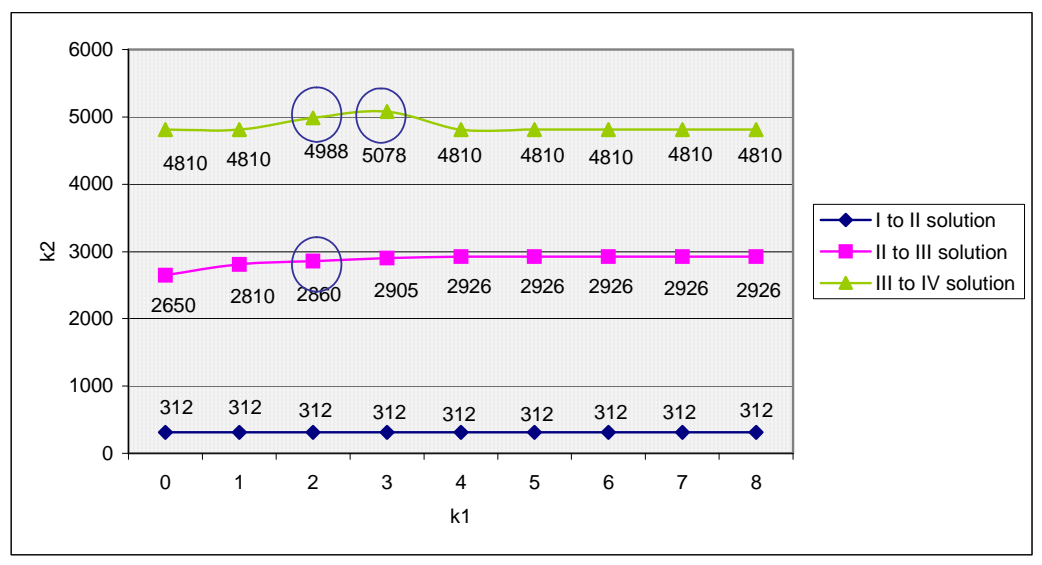

There are 2 types of third solution and 3 types of fourth solution that appear in the analysis when the values of two penalties $k_{1}$ and $k_{2}$ are varying and it is presented in Table 5 and Table 6.

The structures of solutions III and IV are changed because the penalty cost for each minute of delay $\left(k_{1}\right)$ increased sufficiently to justify the use of the larger aircraft (aircraft with higher operational costs) to execute the affected flights. 
Table 5 The presentation of III solution

\begin{tabular}{|c|c|c|}
\hline Penalty $\mathbf{k}_{\mathbf{1}}$ & I I I solution & \\
\hline 0 & IV solution & \\
\hline 2 & $\begin{array}{l}\text { - Priority flights OS301 and OS302 are reassigned to aircraft A320 (OELBO) and are } \\
\text { executed with delay (delay on flight OS301 is } 162 \text { min and } 111 \text { min on flight OS302). } \\
\text { - Non-priority flights OS313 and OS314 are cancelled. }\end{array}$ & 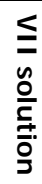 \\
\hline
\end{tabular}

Table 6 The presentation of IV solution

\begin{tabular}{|cc|}
\hline Penalty $\mathbf{k}_{\mathbf{1}}$ & IV solution \\
\hline 0 & Basic solution \\
\hline 2 & V solution \\
\hline 3 & VI solution \\
\hline
\end{tabular}

The last proposed IV solution is equal to the previously attained "optimal" solution for $k(i)=1952$ and $k_{1}=3$. Based on this, the previous statement of gaining the "optimal" solution can be improved and is worded like this: for gaining the "optimal" solution the $k_{1}$ value should not be less then 3 , the $k$ (i) value should not be less then 1952 and $k_{2}$ should not be less then 5078 (or $k_{1}>4$ and $k_{2}>4810$ ).

Figure 8 The airline's profit regarding to penalties values, $k_{1}$ and $k_{2}$

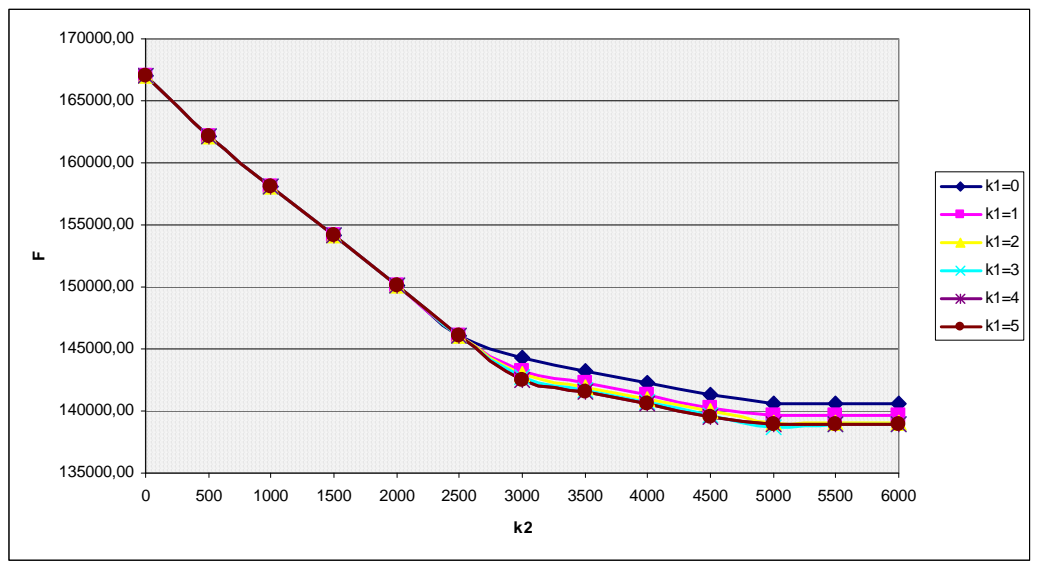


In Figure 8 it can be seen that while the values of $k_{1}$ and $k_{2}$ increase, the value of airline's profit decreases down to its minimum (until the value that has in IV solution) and after that is constant. Figure 8 also shows the airline's profit sensitivity to penalty value changes of $k_{1}$ and $k_{2}$ and it can be noted that the value of $k_{2}$ has influence on the slope of the function till the value of 5000 , but the value of $k_{1}$ determined its value. Meaning, that increasing the value of $k_{1}$ will decrease the airline's profit more and more i.e. the airline's profit will have lower minimum and it will reach that minimum at higher values of $k_{2}$.

\subsubsection{PENALTY FOR PRIORITY FLIGHT CANCELLATION k(i) AND PENALTY FOR NON- PRIORITY FLIGHT CANCELLATION $k_{2}$}

The same procedure was applied on the last pair of penalties $k(i)$ and $k_{2}$. The common element for all phases is that in each phase the overall number of different solutions is 4, regardless of the value of $k(i)$. Also, the first and the second solution are the same in all phases as well as the value where the solution change occurs from the first to the second. The structure of the third and the fourth solution vary through phases, but the value where the solution change occur from III to IV is always the same.

The structures of the first and the second solutions are equal to I and II solution in the previous analysis (pair of penalties $k_{1}$ and $k_{2}$ ). Figure 9 shows the point where solution change occurs and how the value of this point $\left(k_{2}\right)$ changes in regards to changing the value of penalty $k(i)$. According to the fact that each phase has 4 solutions, Figure 9 presents the value of penalty $k_{2}$ where solution change occurs from I to II solution, from II to III solution and, finally, from III to IV solution. 
Figure 9 Changing the point of solution replacement regards to changing the value of penalty $k(i)$

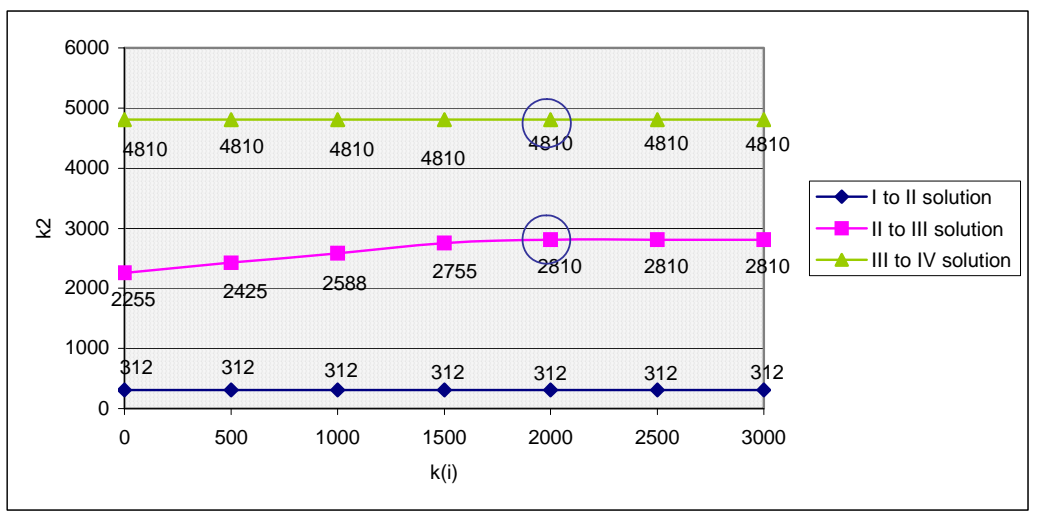

Also indicated is where the structure of solutions III and IV changes, for an increasing value of $k(i)$. There are 2 types of third solutions and 2 types of fourth solutions that appear in the analysis where the values of two penalties, $k(i)$ and $k_{2}$, vary. These solutions are presented in Table 7 and Table 8.

Table 7 The presentation of III solution

\begin{tabular}{|c|c|c|}
\hline Penalty k(i) & III solution & \\
\hline 0 & - Flights OS301, OS302, OS313 and OS314 are cancelled. & 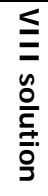 \\
\hline 2000 & IV solution & - \\
\hline
\end{tabular}

Table 8 The presentation of IV solution

\begin{tabular}{|cl|}
\hline Penalty k(i) & IV solution \\
\hline 0 & I solution \\
\hline 2000 & Basic solution \\
\hline
\end{tabular}


The structures of III and IV solutions are changed because the penalty cost for priority flight cancellation, $\mathrm{k}(\mathrm{i})$, increased enough to justify the use of another aircraft to execute the affected priority flights, as well as moving the departure time of certain flights for a later time. As a result, instead of 4 cancelled flights, all flights are executed on time or with allowed delay.

The results from Figure 10 show that while the values of $k(i)$ and $k_{2}$ increase, the value of airline's profit decreases down to its minimum (until the value that has in the IV solution) and after that is constant. It also shows the airline's profit sensitivity to penalty value changing of $k$ (i) and $k_{2}$. For $k_{2}$ values lower then 2000 the airline's profit is very sensitive, then between the values 2000 and 5000 the sensitivity is lower and for the values higher then 5000 the airline's profit is insensitive. Also, increasing the value of $k(i)$ will increasingly decrease the airline's profit. The penalty values of $k(i)$ have no influence on the airline's profit for values of $k_{2}<2000$ because all flights in solution are executed on time or cancelled. There are no delayed flights.

Figure 10 The objective function values in regard to penalty values, $k(i)$ and $k_{2}$

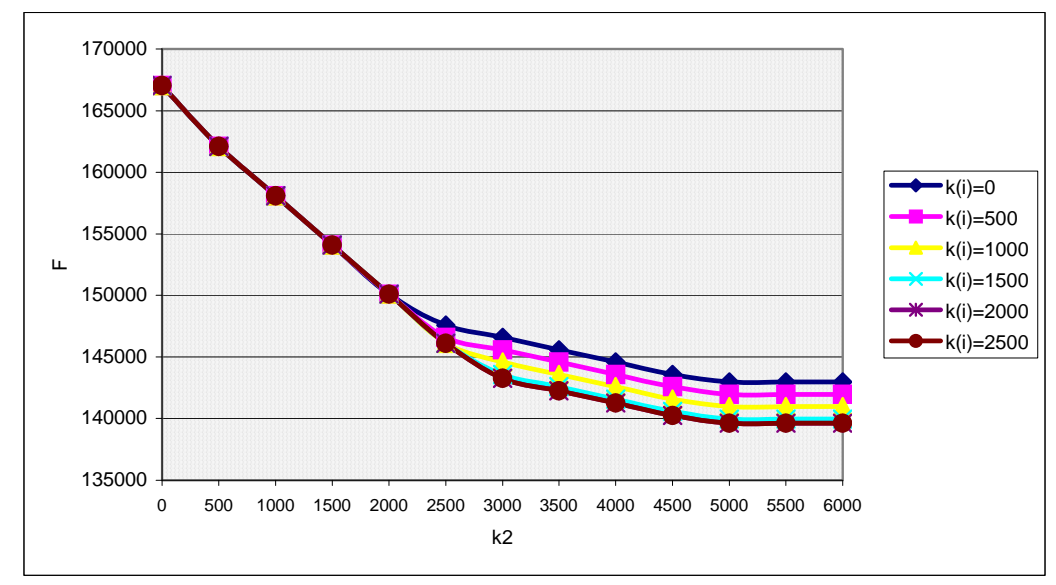

\section{CONCLUSION}

The ASO Advanced model has been developed as a decision support system and its goal is, in situations when a carrier's scheduled activities are disturbed, to provide a solution within a short period of time, which would minimize the negative effects of these disruptions. This problem is very complex and requires precise definition of all criteria, assumptions and operational constraints so that the proposed solution is compliant with the airline's goals and preferences. 
Since certain parameters (penalties) used in this model are difficult to measure, the sensitivity analysis of the ASO Advanced model to these penalties is one way that can help in their determination. The presented sensitivity analysis is also useful for determining the "optimal" solution or set of "optimal" solutions which best represent the carrier's interests and also to determine the range of penalty intervals within the solution is the same.

The results from the first sensitivity analysis, where only one penalty value is changed while the rest of the penalties have fixed, assumed values, are useful for gaining the values which will lead to logical and expectable solutions. All the considered penalties have lower limits than the assumed one, except the penalty $k_{1}$ which can have any value depending on current airline's policy. This conclusion is based on solutions proposed by the ASO Advanced model where for each assumed value of $k_{1}$ the generated solution is acceptable. The only difference is that in some solutions the affected flights are executed with a delay by an aircraft with lower or equal operational costs, then broken one and in some solutions the affected flights are executed on time by aircraft with higher operational costs then broken one.

The results from the second sensitivity analysis, where two penalties values are changed while the rest of the penalties have fixed, assumed values, are useful for gaining the values which will lead to "optimal" solution i.e. to the best solution from the aspect of delay and cancellation. The values for gaining the "optimal" solution are: the $k_{1}$ value should not be less then 3 , the $k(i)$ value should not be less then 1952 and $k_{2}$ should not be less then 5078 (or $k_{1}<4$ and $k_{2}<4810$ ). This solution can be considered as the best one from the aspect of delay and cancellation cause in the situation where one aircraft is out of order 12 hours and there is a possibility of cancelling certain flights, still exists a solution where all planned flights can be executed on time with the available fleet.

\section{ACKNOWLEDGEMENTS}

The paper presents extended work on the Airline Schedule Optimization project and Project 15023, sponsored by Serbian Ministry of Science and Technological Development. 


\section{References}

- Jarrah, A. I., Yu, G., Krishnamurthy, N., Rakshit, A., (1993) A Decision Support Framework for Airline Flight Cancellations and Delays. Transportation Science, Vol. 27, No. 3, pp. 266280.

- Krcevinac, S., Cangalovic, M., Kovacevic-Vujcic, V., Martic, M., Vujocevic, M., (2004) Operational Research, Faculty of Organizational Sciences University of Belgrade.

- Nedeljkovic S., Jakimovska V.,. Babic O, Cangalovic M., (2004) Daily Operational Flight Schedule Design. Proceedings SYM-OP-IS 2004 (XXXI Symposium on Operations Research), Fruska Gora, Serbia and Montenegro, pp. 233-238.

- Institute of Faculty of Transport and Traffic Engineering, Belgrade, (July 2002 - January 2005) Airline Schedule Optimization. Clients: Ministry of Science, Technology and Development of Republic of Serbia and Yugoslav Airlines as participant.

- Thengvall, B., Bard, J., Yu, G., (2000) Balancing User Preferences for Aircraft Schedule Recovery during Irregular Operations, IIE Transactions 32, pp. 181-193. 